\title{
UNINPSl:
}

\section{cerca de las familias en la incertidumbre}

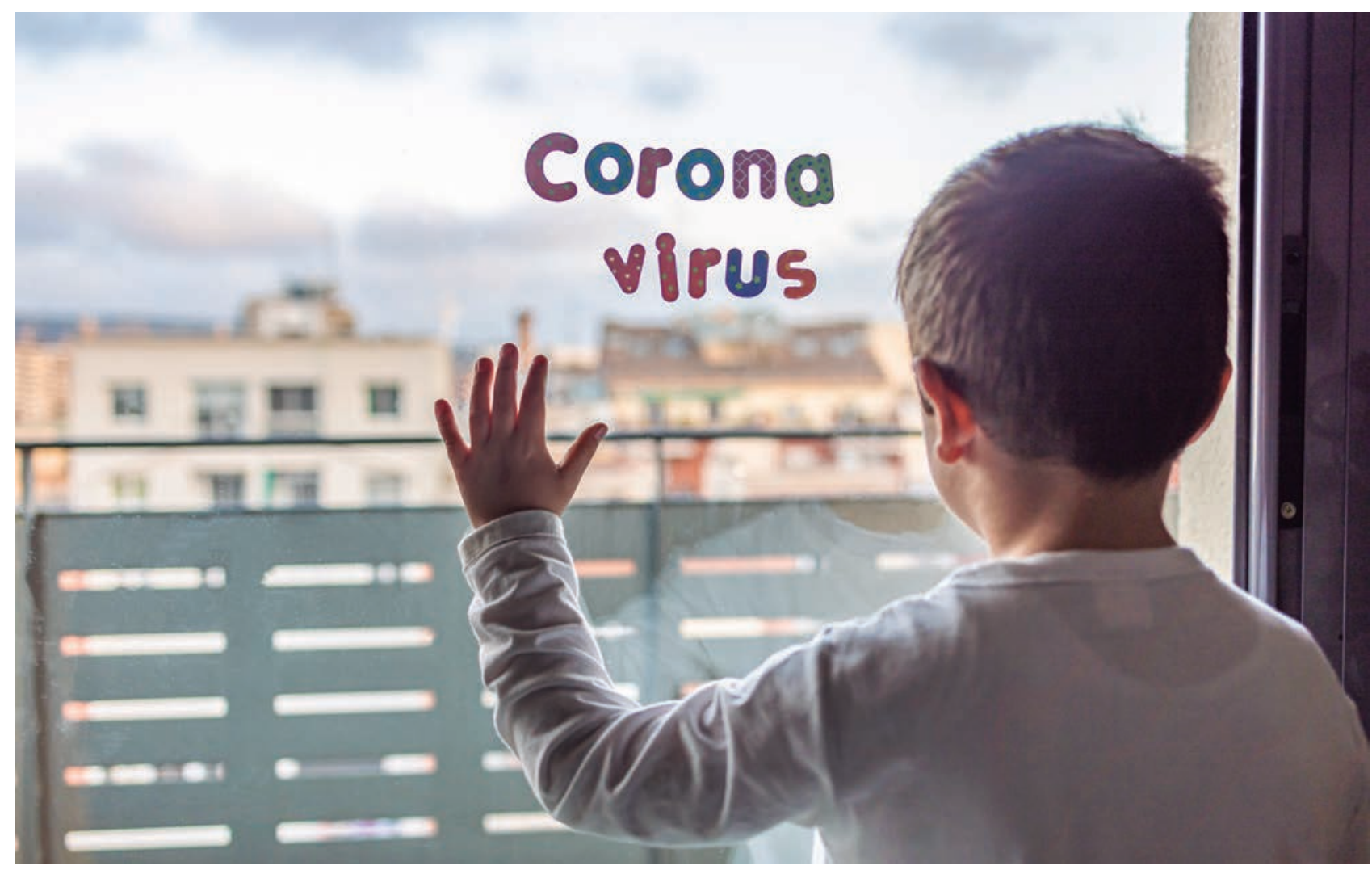

Este artículo presenta la experiencia de acompañamiento a las familias llevada a cabo por los profesionales de la Unidad Clínica de Psicología UNINPSI de la

Virginia Cagigal de Gregorio Universidad Pontificia Comillas vcagigal@comillas.edu Universidad Pontificia Comillas, ante la crisis de la COVID-19, acompañamiento centrado en los miedos, la vulnerabilidad, los duelos, la tensión y estrés de la intensa convivencia en casa, los ajustes de pareja y la resiliencia familiar. Se destaca la capacidad de afrontamiento positivo por parte de una gran mayoría de las familias atendidas. 


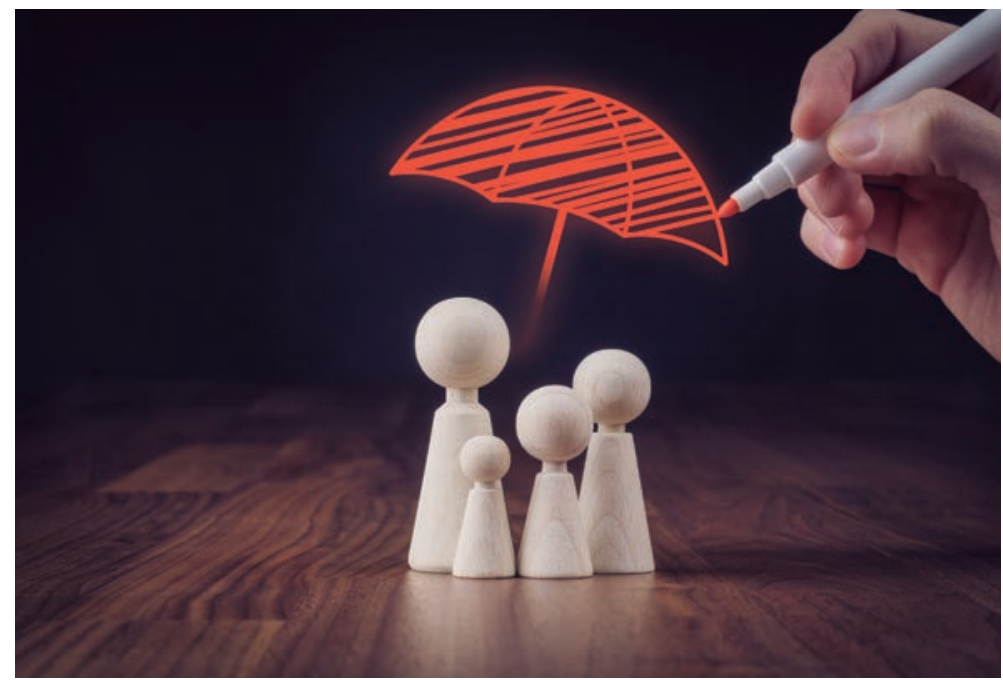

La declaración del estado de alarma en España con motivo de la crisis de la COVID-19 en marzo de 2020 ha sumido a muchas familias en una situación de incertidumbre y dolor, en una realidad nunca antes experimentada. Si bien en los primeros momentos pudo parecer que se trataba de algo transitorio, pasada la Semana Santa ya las familias experimentaban el peso de lo que comenzaba a vislumbrarse como duradero en el tiempo, y costoso de sostener en muchos sentidos. Además, en Madrid se habían clausurado los colegios una semana antes, lo que añadía tiempo de ajuste y tensión familiar.

Ante esta transformación social provocada por el coronavirus, todos los profesionales de la Unidad Clínica de Psicología UNINPSI de la Universidad Pontificia Comillas se volcaron en responder de la manera más humana, profesional y ajustada posible a las necesidades de todos los pacientes, fueran adultos, familias, parejas, niños o adolescentes. Esto ha supuesto que, no solamente no se ha rebajado el número de intervenciones en relación con los años anteriores, sino que incluso se han incrementado, sobrepasando por primera vez el umbral de las 10.000 sesiones anuales atendidas en este centro; de ellas, más de 2000 intervenciones han sido específicamente con niños, adolescentes y familias* .

Pero los números no son los que mejor dan cuenta de cómo se ha querido seguir atendiendo a tantas personas, sino que hemos de analizar las líneas que están marcando esa tarea de acompañar, en este caso a las familias, a los padres, a los hijos, a los abuelos, con la vocación de seguir siendo herramienta de soporte y sostén para tantos.

La labor de los psicólogos clínicos y sanitarios permite entrar en territorios sagrados para las personas; con el permiso de los propios pacientes, podemos adentrarnos en los rincones de dolor, de desesperanza, de angustia, y ser testigos del esfuerzo por vencer lo difícil, por superarse a uno mismo... Es tarea privilegiada, que suscita en el corazón del profesional el asombro por la capacidad

* Este artículo se centra en la experiencia del acompañamiento a través de la terapia con familias desde la UNINPSI. Además de continuar con la labor terapéutica, en colaboración con la Provincia de España de la Compañía de Jesús, el 20 de marzo de 2020 pusimos en marcha un servicio de escucha telefónica para profesionales sanitarios www.sanitarios.noestassolo.es, desde el que se ha dado escucha y apoyo emocional a más de cien profesionales durante el estado de alarma. El Equipo de Vida religiosa de la UNINPSI elaboró el documento Orientaciones para el cuidado psicológico durante el tiempo de cuarentena en la vida religiosa y sacerdotal, traducido a diversas lenguas y difundido a comunidades y diócesis de muy diferentes lugares del mundo. Asimismo, en colaboración con profesores del Departamento de Psicolo. gía y del Instituto Universitario de la Familia, se desarrolló una encuesta online, iCygnus, para ofrecer orientaciones a los padres que ayudaran en el tiempo de confinamiento; este mismo equipo desarrolló otra encuesta, iEncuist, para ofrecer claves a personas adultas.

Un equipo formado por más de treinta profesores e investigadores de las diferentes facultades de la Universidad Pontificia Comillas y de la UNINPSI puso en marcha el Proyecto Gaudete, con el objetivo de ofrecer líneas de esperanza, sentido y discernimiento para responder a la crisis de la COVID-19 (https:// proyectogaudete.com/).

En la actualidad, de forma gratuita gracias al patrocinio de Mylnvestor, S. L., en la UNINPSI se están llevando a cabo grupos de apoyo psicológico para profesionales sanitarios, terapias individuales, familiares o de pareja para profesionales sanitarios, así como el curso Cuida y cuídate, sobre autocuidado en situaciones de crisis y emergencias para profesionales sanitarios (quienes estén interesados en estos proyectos pueden contactar con uninpsi@comillas.edu). 
de lucha del ser humano y admiración por la persona que se esfuerza por salir adelante, a veces con tantos elementos en contra. Y la crisis del coronavirus evidentemente nos ha dejado huella de muchas de estas batallas personales y familiares, que hemos podido acompañar.

Hemos acompañado y seguimos acompañando los miedos: miedo a lo desconocido, miedo a la enfermedad inesperada e inexplicada, miedo a las pérdidas de seres queridos, miedo a no poder despedir, miedo de los padres a transmitir su miedo a los hijos, miedo a las consecuencias económicas de las pérdidas del trabajo en las familias, miedo a contagiar a los cercanos (especialmente en los profesionales sanitarios y de profesiones esenciales). Ha sido y está siendo un tiempo de miedo, porque nos estamos enfrentando a un enemigo desconocido, lo que ha supuesto informaciones y medidas contradictorias. Vivimos acontecimientos que más parecen sacados de una terrible película de ciencia ficción que hechos reales. Si meses antes nos hubieran contado lo que íbamos a vivir, difícilmente hubiéramos dado crédito.

Acompañar los miedos supone darles legitimidad: "Claro que es comprensible e incluso esperable que, ante esto, tengas miedo", lo que permite que la persona no se sienta torpe, inútil, incapaz de autogestionarse. Acompañar los miedos también implica poderlos colocar en un lugar no excesivamente invasivo, ayudando a ganar cierto control sobre ellos: "Es lógico sentir esto, pero vamos a ponerle orden para que no te invada todo el día". En el caso de los padres, acompañar sus miedos requiere trabajar con ellos los límites en casa: "cuántas veces al día vamos a ver las noticias", "cuánto vamos a exponer a los hijos a conversaciones sobre las circunstancias tan terribles que estamos viviendo", "en qué medida y de qué modo les vamos a contar a los hijos noticias difíciles sobre la familia, enfermedades, el trabajo...". Estar cerca de las familias nos ha permitido ser testigos de cómo iban dominando los miedos o cómo los iban gestionando mejor, cómo han ido
Acompañar los miedos

\author{
Legitimar \\ Ganar control \\ Poner límites
}

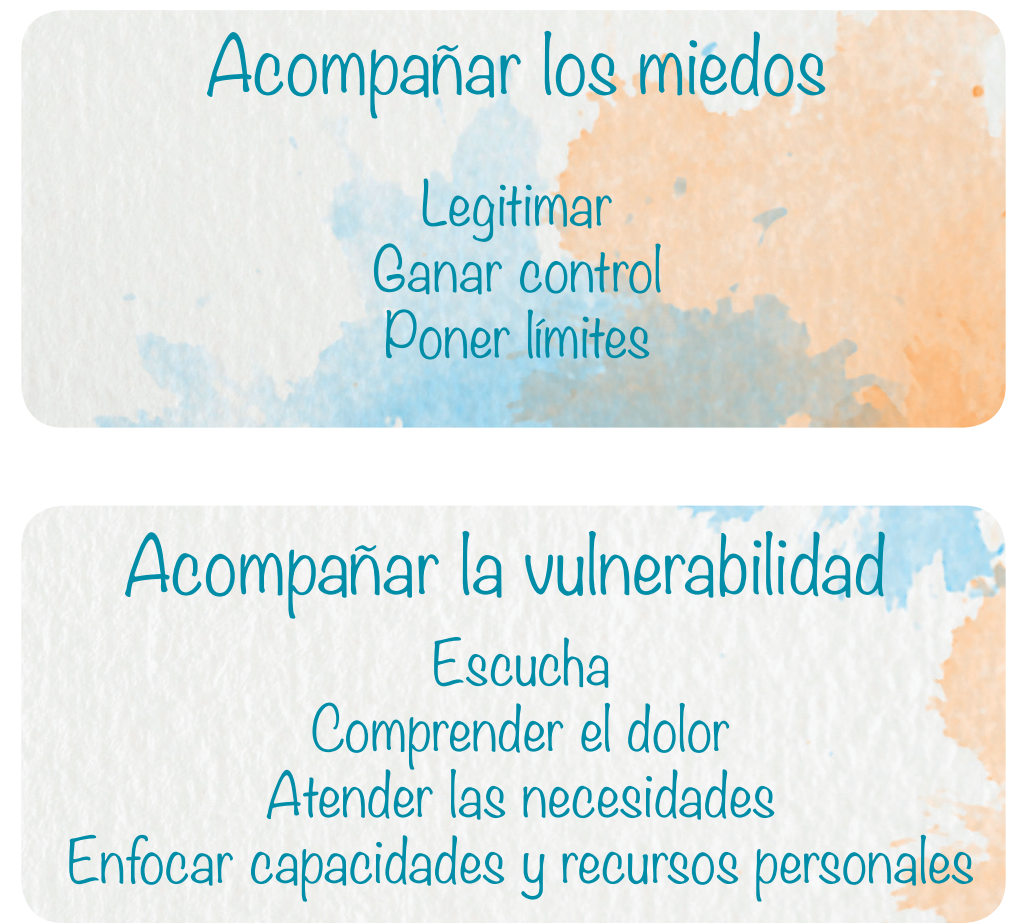

dando sentido a su experiencia y logrando un cierto control, aunque lo externo no mejorara. Hemos visto a muchas personas manejar las situaciones, ganando en calma interior.

Hemos acompañado y seguimos acompañando la vulnerabilidad. Nuestra sociedad, que creíamos preparada para no ser tambaleada por nada, de pronto ha palpado la vulnerabilidad. Y nos hemos percatado de que no podemos con todo ni somos inmunes a todo. Además, la vulnerabilidad ha hecho todavía más vulnerables a los vulnerables. Lo saben bien en muchos centros escolares, en los que han hecho grandísimos esfuerzos para llegar a tantas familias sin recursos para comer - para hacer llegar soportes informáticos, para que los hijos pudieran seguir las clases. También en la UNINPSI hemos visto crecer la demanda de apoyo económico para las intervenciones y vamos abordando con algunos padres y madres cómo reorientar su desarrollo profesional, amortiguando no pocas veces el impacto negativo de la pérdida de estatus o la quiebra de una carrera profesional hasta el momento bien encarrilada.

Acompañar la vulnerabilidad implica, sobre todo, escuchar en profundidad a la persona, asomarse al mundo que se le ha caído, entender su dolor ante la inseguridad provocada y atender su necesidad. Implica también ayudarle a enfocar 


\section{IIII. Â乌ORA DE PROFESORES}

Tras tantas horas extra y desvelos en todo este tiempo de pandemia, no siempre se reconoce suficientemente el trabajo de los profesores, no parece que la sociedad haya hecho explícito con la claridad necesaria el agradecimiento por todo ese cuidado a los niños y jóvenes de tantos rincones del país, por toda la dedicación y el mimo que tantos maestros han puesto para dar lo mejor de sí y amortiguar lo más posible el efecto de tanto tiempo alejados de las aulas.

Esto puede poner en riesgo la propia implicación en la tarea, Ilevando al desánimo 0 a una cierta quemazón (burn ouf). Una ayuda para afrontar esta vivencia y no sumirse en el desaliento es recuperar sentido a lo que se hace: ¿cuál es el objetivo de mi día a día con los alumnos? ¿Hacia dónde se dirige mi mirada cuando pienso cómo deseo ser como profesional, cómo deseo dibujar mi perfil de profesor o profesora? Si pienso en que algún alumno me recuerde cuando sea mayor, ¿qué herencia, en términos de valores, creo que estoy dejando a mis alumnos? ¿Qué les ha quedado a mis alumnos de mí como referente en este tiempo de confinamiento? ¿Soy una figura de apoyo para las familias en sus circunstancias difíciles? ¿Qué me gustaría transmitirles de mi forma de ser y estar como maestro o maestra en esta etapa compleja, llena de límites espaciales, límites al contacto, límites a la cercanía? lo que ocurría y, por tanto, sin poder graduar el impacto, por sorpresa, sin poder prepararse. Además, muchas veces han estado rodeadas de incertidumbre, de no saber qué le estaría pasando a la persona querida que estaba en el hospital o en la residencia; y, en consecuencia, han generado indefensión por no poder hacer nada. Además, la falta de información no permitía entender lo que sucedía. Muchas han sido pérdidas en soledad, soledad para quien estaba enfermo, para quien fallecía, y soledad para quien quedaba, que ni siquiera después podía recibir el abrazo consolador y reparador de los seres queridos. Todo ello ha tenido como consecuencia una resonancia emocional muy intensa, de impotencia, culpa, desbordamiento.

Acompañar en los duelos implica estar cerca, hacerse presente, respetar, dejar que sea el alma dolorida la que busque y encuentre el apoyo en el momento en que lo necesite, sin invadir y sin alejarse. Supone también promover el autocuidado, sabiendo que, aunque la infinita tristeza invite a abandonarse, sin embargo, uno merece cuidarse a sí mismo, ser delicado consigo mismo, tomando conciencia de que quedan otros que lo necesitan. Acompañar en los duelos en la circunstancia específica de la crisis del coronavirus nos ha llevado a ayudar a las familias a crear sencillos rituales en las casas para poder despedirse de los seres queridos: "tanatorios virtuales" para poder compartir con los más allegados que físicamente no podían juntarse, cartas de agradecimiento por la vida de la persona que nos ha dejado, rincones de recuerdos y homenaje... Y hemos sido testigos de familias que han dado espacio a compartir y hablar sobre la persona querida, han reído y llorado juntos, han podido perdonar al que se ha ido si había alguna cuestión pendiente o sentir su perdón simbólico, han podido expresarse el cariño, respetando también los tiempos de cada uno y las diferencias en las generaciones a la hora de vivir la pérdida, han podido recoger el legado recibido de la persona que se ha marchado. 


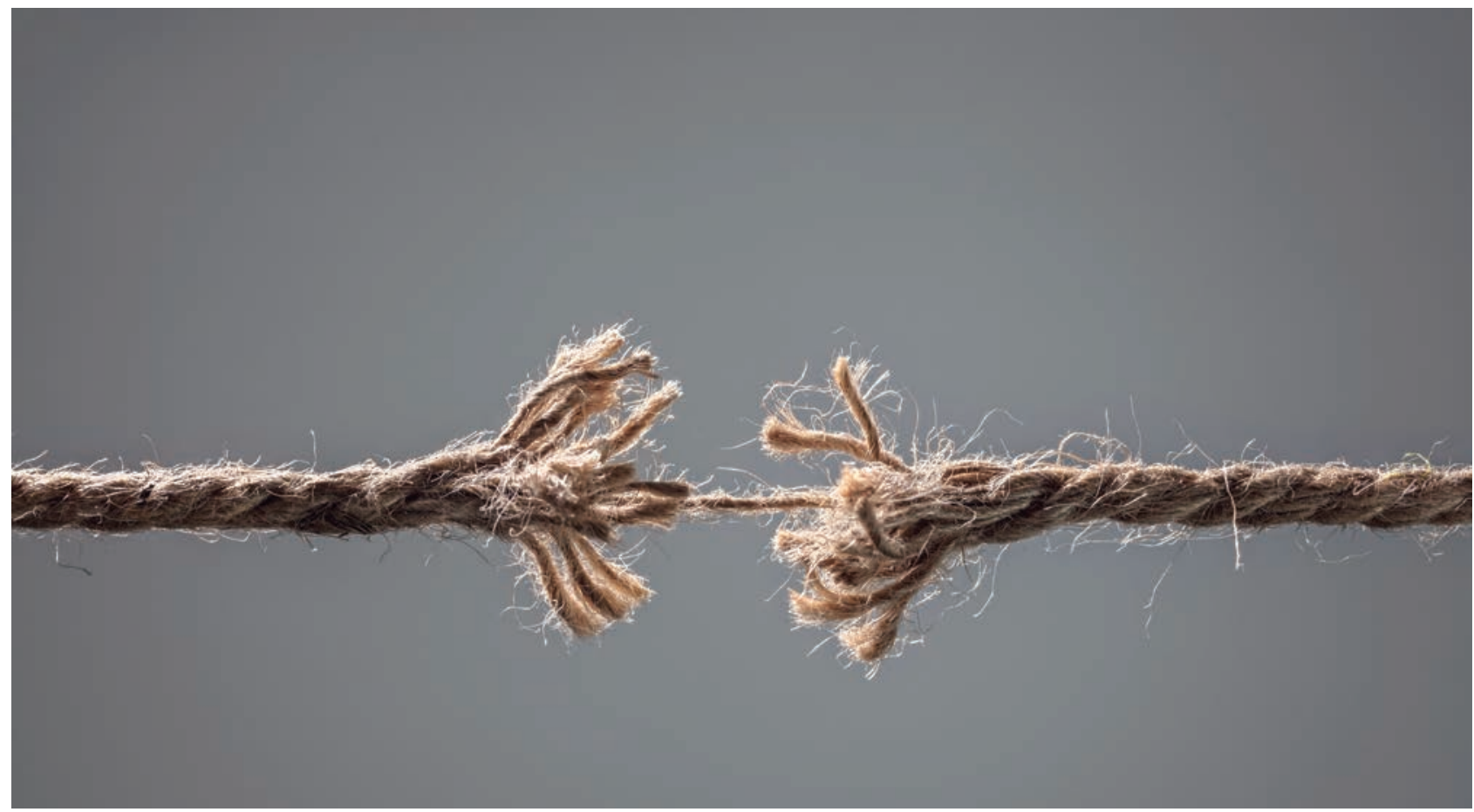

Hemos acompañado y seguimos acompañando la tensión y el estrés familiar de acomodarse a la convivencia en casa. El confinamiento supuso pasar de unas vidas volcadas en bastante medida hacia el exterior del hogar (trabajos, colegios, universidad, reuniones con amigos, quedadas, deportes en el exterior, aficiones, ocio...) a un cien por cien en el hogar. Para la mayoría de los padres ha sido muy difícil combinar el teletrabajo con la atención a los hijos; además es difícil replantearse el ocio en casa sin que pase por las pantallas y las tecnologías. Ha habido que organizar los horarios, tratar de establecer rutinas que permitieran diferenciar la semana del fin de semana, coordinarse para repartir los tiempos con los hijos y los tiempos profesionales, regularse para resolver los conflictos en unos pocos metros cuadrados sin poder salir.

Acompañar la tensión y el estrés de la intensa convivencia en casa ha supuesto ayudar a que estén atentos unos a otros y comprendan sus diferentes necesidades. También conlleva promover flexibilidad, imprescindible para ajustarse a circunstancias tan novedosas y no vividas antes, puesto que no es posible seguir haciendo como si no pasara nada: ha sucedido una verdadera transformación en nuestras vidas, a la que, si se responde de una forma elástica, es mucho más posible acomodarse que si se responde con rigidez.
Se trata de una flexibilidad compleja porque a su vez las familias han necesitado, por una parte, poner orden en horarios de sueño, comidas, estudios, espacios y, por otra, ha sido importante dar peso a la autoridad de los padres. Para los padres, se ha añadido el esfuerzo de convertirse en el principal apoyo del aprendizaje escolar de sus hijos, al tiempo que la situación ha exigido de los profesores horas interminables de trabajo y grandes esfuerzos de adaptación a la nueva dinámica de enseñanza-aprendizaje. Hemos sido testigos de cómo mucha familias han ido negociando, entre la pareja, o padres e hijos, para ir encontrando ese equilibrio
Para algunas parejas ha sido un tiempo de honda crisis, al caer en la cuenta de sus lagunas, sus agujeros, sus déficits

\title{
Acompañar la tensión y el estrés familiar de la convivencia
}

\author{
Promover comprensión de unos a otros \\ Flexibilizar \\ Reorganizar tiempos \\ Poner orden \\ Mantener autoridad
}




\section{Acompañar los ajustes de relación de pareja}

Darse tiempo para recuperar y reconstruir Eliminar prejucios sobre el otro Apreciar lo nuevo del otro Mejorar la comunicación Clarificar roles Expresar cariño y amor Poner límites para preservar la intimidad para estar a gusto en casa, atendiendo a cómo cada uno necesita gestionar sus tiempos personales, sus tiempos compartidos, sus diferentes actividades; hemos visto a familias en habitaciones pequeñas de casas pequeñas dialogar para organizarse y compartir los lugares, los tiempos, las actividades; Ios hemos visto reorganizarse en las tareas de la casa, las compras, los cuidados de los más pequeños y de los mayores.

Hemos acompañado y seguimos acompañando los ajustes de pareja. De pronto, marido y mujer han compartido más tiempo que nunca, han pasado más horas en el espacio físico de la casa, sumando casi más horas que todas las que Ilevan juntos en su vida en común. Y esto, a veces, ha puesto en evidencia la falta de complicidad, la ausencia de vida en común, las diferencias en gustos, ocio, necesidades, intereses... No es fácil lidiar con esos descubrimientos sobre todo cuando, además, no se puede apoyar la resolución de estas dificultades apoyándose en el exterior. Para algunas parejas ha sido un tiempo de honda crisis, al caer en la cuenta de sus lagunas, sus agujeros, sus déficits.

Acompañar los ajustes de la pareja en una situación de confinamiento supone facilitar que los cónyuges se den tiempo para recuperar, para reconstruir o para construir algo nuevo. Supone ayudarles a eliminar juicios y prejuicios sobre el otro, dando oportunidad para que puedan apreciar algo nuevo en el otro, puedan mirarse con ojos no viciados por lo histórico, puedan acercarse a descubrir algo diferente, deseen ofrecerse uno a otro algo distinto, puedan reinventar su vida de pareja. Para acompañar a las parejas en esta situación hemos abordado la comunicación, la definición de sus roles, la expresión del cariño, del afecto, del amor; hemos trabajado para ayudarles a delimitar lo que es de la pareja y lo que es de la familia, poniendo límites claros que ayuden a preservar y dar valor a lo singular de su vida íntima de pareja adulta. Hemos visto cómo ha habido parejas que se han vuelto a mirar a los ojos, se han vuelto a sentir necesarios el uno para el otro, han recuperado alegría de vivir juntos, han dado nuevo valor a lo que cada uno le aporta al otro.

Durante todo este tiempo de pandemia, hemos querido y queremos seguir acompañando la resiliencia familiar: creemos que es fundamental, a pesar de las duras adversidades que se están viviendo, promover una respuesta resiliente en las familias, que supone poder adaptarse y desplegar recursos ante la adversidad, rescatando la capacidad de afrontar que tenemos los seres humanos e incluso, si es posible, poder salir de ello habiendo crecido interiormente. Para construir la resiliencia, se necesitan relaciones íntimas y seguras. Las heridas psicológicas pueden dejar heridas relacionales, porque la persona dañada puede sentir que nadie le va a poder comprender y esa misma experiencia incrementa la vivencia de soledad y lejanía de los seres queridos. Cuando se viven experiencias traumáticas el mundo se vuelve caótico, incoherente, imprevisible, amenazador. La familia muy herida puede replegarse sobre sí misma, experimentando dificultad para hablar de lo doloroso (no hay palabras para expresarlo), cuando además las figuras de seguridad en la familia están también tocadas.

Acompañar la resiliencia familiar implica favorecer espacios para poder compartir la experiencia (no necesariamente espacios catárticos, que tienen el riesgo de abrir una caja de truenos que luego no se puede contener), promoviendo la acogida del sufrimiento del otro, de su rabia, de su culpa, de su tristeza, en un clima de protección, de cobijo, de amparo, validando los sufrimientos y los esfuerzos de ajuste o de cambio, aunque no sean 
todavía muy exitosos. Es necesario dar tiempo para que cada miembro de la familia - diferentes entre sí y cuyas experiencias han sido necesariamente distintas, aunque el hecho acontecido sea el mismo- pueda ir expresándose con libertad y de forma genuina. Para promover la resiliencia familiar, se puede dar significado a lo vivido, integrando las experiencias traumáticas, ofreciendo un apoyo con continuidad tras la urgencia (a veces estamos muy cerca y disponibles en los momentos del pico de la crisis, pero luego no somos capaces de sostener esa cercanía y disponibilidad en el tiempo, siendo sin embargo muy necesario en una fase posterior cuando ya no parece haber tanta urgencia).

Desde nuestra labor de apoyo y acompañamiento terapéutico, estamos viendo a muchas familias descubrir capacidades y recursos con creatividad, sacando energía de donde ya parecía que no había más, remontando una y otra vez (una pérdida, un negocio que se hunde, un trabajo que se pierde...); vemos cómo hay padres que están dando espacio a escuchar a sus hijos, y los ayudan a elaborar una narrativa de lo que está aconteciendo no hiriente, ni acusadora, sino esperanzadora, con perspectiva de futuro, de ajuste a una vida que requiere esforzarse para salir adelante. Hay familias que están logrando un clima sereno a pesar de la intemperie y la reciedumbre de lo que están viviendo.

Durante esta etapa crítica desde la declaración del estado de alarma en nuestro país hasta los días de incertidumbre actuales, los profesionales de la UNINPSI nos sentimos agradecidos por todo lo que estamos recibiendo de las personas que nos están abriendo sus hogares (ahora

\section{HEMOS HABLADO DE}

\section{Familia; afrontamiento; resiliencia.}

Este artículo fue solicitado por PADRES Y MAESTROS en mayo de 2020, revisado y aceptado en octubre de 2020.

\section{Acompañar la resiliencia familiar}

Compartir las experiencias personales Acoger el sufrimiento Promover protección Validar el sufrimiento

Dar tiempo a cada uno para expresar su vivencia

\section{Dar significado a la experiencia promover recursos}

más que nunca, ya que muchas veces nos conectamos online) y el corazón de su familia, regalándonos el privilegio de ser testigos de cómo el ser humano es capaz de superar las dificultades y de convertir las experiencias dolorosas en motivos para crecer y para superarse, tal como reflejaba una niña entrevistada en televisión, que traducía esta experiencia de forma preciosamente ingenua y positiva cuando decía que le estaba gustando mucho el confinamiento porque pasaba mucho más tiempo con papá y con mamá. A los niños y a los adolescentes les calma la proximidad serena de sus padres y sus seres de referencia; las experiencias duras tienen mucha menos probabilidad de convertirse en traumas para ellos si pueden sentirse sostenidos y abrazados por un entorno de cariño, tranquilidad emocional y disponibilidad. Aunque quisiéramos que esto nunca hubiera pasado, sin embargo, es un privilegio poder acompañar a tantas familias en una etapa tan crítica como la que estamos viviendo. Ojalá que juntos seamos capaces de seguir abriendo puertas de esperanza •

\section{DARA SABER MÂS}

Delage, M. (2014). La resiliencia familiar. El vínculo familiar y la superación de las heridas. Barcelona: Gedisa.

FMLC. Fundación Mario Losantos del Campo, especializada en duelo. [Archivo de blog]. Recuperado de https://www.fundacionmlc.org/ blog/

VV. AA. Consejos psicológicos para largos periodos dentro de casa. Recuperado de https://drive.google.com/drive/u/0/folders/1u1PoxURIdkPvP1. 07wvZ5f_S04qOGezm 